Benlian Lv* and Yunli Ren

\title{
Crystal structure of poly[diaqua- $\left(\mu_{2^{-}}\right.$ 2,5-dihydroxyterephthalato- $\left.\mathrm{K}^{2} \mathrm{O}: 0\right)\left(\mu_{2^{-}}\right.$ bis(4-pyridylformyl)piperazine- ${ }^{2} N: N^{\prime}$ ) cadmium(II)] dihydrate, $\mathrm{C}_{24} \mathrm{H}_{28} \mathrm{CdN}_{4} \mathrm{O}_{12}$
}

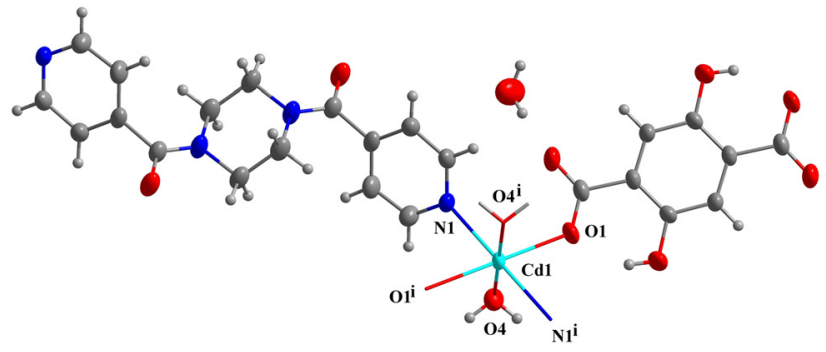

https://doi.org/10.1515/ncrs-2021-0167

Received May 3, 2021; accepted May 31, 2021;

Published online June 18, 2021

\begin{abstract}
$\mathrm{C}_{24} \mathrm{H}_{28} \mathrm{CdN}_{4} \mathrm{O}_{12}$, monoclinic, $P 2_{1} / c$ (no. 14), $a=11.0801(4) \AA$, $b=8.3925(3) \AA, c=13.9503(5) \AA, \beta=102.810(3)^{\circ}, Z=2$, $V=1353.89(8) \AA^{3}, R_{g t}(F)=0.0273, w R_{r e f}\left(F^{2}\right)=0.0633$, $T=291 \mathrm{~K}$.
\end{abstract}

CCDC no.: 2081447

Table 1 contains crystallographic data and Table 2 contains the list of the atoms including atomic coordinates and displacement parameters.

\section{Source of material}

A mixture of $\mathrm{Cd}(\mathrm{OAc})_{2} \cdot 2 \mathrm{H}_{2} \mathrm{O}(26.7 \mathrm{mg}, 0.1 \mathrm{mmol})$, 2,5-dihydroxy-1,4-benzenedicarboxylic acid $(18.9 \mathrm{mg}$, $0.1 \mathrm{mmol}$ ), bis(4-pyridylformyl)piperazine $(29.6 \mathrm{mg}$, $0.1 \mathrm{mmol}$ ) and $8 \mathrm{~mL}$ distilled water in a $25 \mathrm{~mL}$ Teflon-lined autoclave was kept under autogenous pressure at $353 \mathrm{~K}$ for four days. After cooling down to room temperature

*Corresponding author: Benlian Lv, School of Chemical Engineering and Pharmaceutics, Henan University of Science and Technology, Luoyang 471023, P. R. China, E-mail: bl9901343@163.com Yunli Ren, School of Chemical Engineering and Pharmaceutics, Henan University of Science and Technology, Luoyang 471023, P. R. China
Table 1: Data collection and handling.

\begin{tabular}{|c|c|}
\hline Crystal: & Colourless block \\
\hline Size: & $0.31 \times 0.28 \times 0.25 \mathrm{~mm}$ \\
\hline Wavelength: & Mo $K \alpha$ radiation $(0.71073 \AA)$ \\
\hline$\mu$ : & $0.88 \mathrm{~mm}^{-1}$ \\
\hline Diffractometer, scan mode: & SuperNova, $\omega$ \\
\hline$\theta_{\max }$, completeness: & $25.0^{\circ}, 99 \%$ \\
\hline$N(h k l)_{\text {measured }}, N(h k l)_{\text {unique }}, R_{\text {int }}:$ & $15330,2347,0.030$ \\
\hline Criterion for $I_{\mathrm{obs}}, N(h k l)_{\mathrm{gt}}$ : & $I_{\text {obs }}>2 \sigma\left(I_{\text {obs }}\right), 2117$ \\
\hline$N(\text { param })_{\text {refined }}:$ & 192 \\
\hline Programs: & $\begin{array}{l}\text { CrysAlis }^{\text {PRO }}[1], \text { SHELX [2, 3], } \\
\text { Olex2 [4] }\end{array}$ \\
\hline
\end{tabular}

naturally, colourless crystals were collected by filtration and washed with distilled water in $32 \%$ yield.

\section{Experimental details}

Hydrogen atoms were placed in their geometrically idealized positions and constrained to ride on their parent atoms.

\section{Comment}

Coordination polymers (CPs), have attracted interests due to their potential applications in gas storage, molecular recognition, catalysis, chromism, and magnetism [5-9]. The structures of CPs based on central metal ions and organic ligands are difficult to predict. Some factors, such as temperature, solvent, $\mathrm{pH}$ value, counterion, metal-to-ligand ratio, etc., have unpredictable impacts in the self-assembly $[10,11]$. From the point of view of structural chemistry, the 2,5-dihydroxy-1,4-benzenedicarboxylate ligand $\left(\mathrm{H}_{2} \mathrm{~L}\right)$ is a good organic linker which may exhibits versatile coordination modes, and useful as a riding ligand $[12,13]$. In this work, we report a $\mathrm{CP}$ based on $\mathrm{Zn}(\mathrm{II})$ ions, $\mathrm{H}_{2} \mathrm{~L}$ and the flexible bis(4-pyridylformyl)piperazine (bpfp) ligand.

The asymmetric unit of the crystal structure consists of one crystallographically independent $\mathrm{Cd}(\mathrm{II})$ centre, one 
Table 2: Fractional atomic coordinates and isotropic or equivalent isotropic displacement parameters $\left(\AA^{2}\right)$.

\begin{tabular}{lrrrr}
\hline Atom & $\boldsymbol{x}$ & $\boldsymbol{y}$ & $\boldsymbol{z}$ & $\boldsymbol{U}_{\text {iso* }} / \boldsymbol{U}_{\text {eq }}$ \\
\hline C1 & $0.7491(2)$ & $0.4829(3)$ & $0.9432(2)$ & $0.0360(6)$ \\
C2 & $0.8789(2)$ & $0.4912(3)$ & $0.97427(19)$ & $0.0297(6)$ \\
C3 & $0.9429(2)$ & $0.5212(3)$ & $0.90396(19)$ & $0.0333(6)$ \\
H3A & 0.9043 & 0.5352 & 0.8388 & $0.040^{*}$ \\
C4 & $1.0618(2)$ & $0.5307(3)$ & $0.92805(19)$ & $0.0327(6)$ \\
C5 & $0.4463(2)$ & $0.5671(4)$ & $0.76476(19)$ & $0.0399(6)$ \\
H5 & 0.5068 & 0.6390 & 0.7835 & $0.048^{*}$ \\
C6 & $0.3837(2)$ & $0.5714(4)$ & $0.66906(19)$ & $0.0408(6)$ \\
H6 & 0.4019 & 0.6441 & 0.6244 & $0.049^{*}$ \\
C7 & $0.2938(2)$ & $0.4659(3)$ & $0.64093(19)$ & $0.0342(6)$ \\
C8 & $0.2718(2)$ & $0.3588(4)$ & $0.7092(2)$ & $0.0433(7)$ \\
H8 & 0.2122 & 0.2851 & 0.6921 & $0.052^{*}$ \\
C9 & $0.3389(2)$ & $0.3622(3)$ & $0.8028(2)$ & $0.0408(7)$ \\
H9 & 0.3236 & 0.2888 & 0.8482 & $0.049^{*}$ \\
C10 & $0.2275(2)$ & $0.4610(3)$ & $0.53671(19)$ & $0.0373(6)$ \\
C11 & $0.0487(3)$ & $0.5070(4)$ & $0.4145(2)$ & $0.0471(8)$ \\
H11A & 0.0921 & 0.4557 & 0.3718 & $0.057^{*}$ \\
H11B & 0.0288 & 0.6138 & 0.3899 & $0.057^{*}$ \\
C12 & $-0.0584(2)$ & $0.4150(4)$ & $0.41435(19)$ & $0.0443(7)$ \\
H12A & -0.1081 & 0.4163 & 0.3490 & $0.053^{*}$ \\
H12B & -0.0387 & 0.3051 & 0.4321 & $0.053^{*}$ \\
Cd1 & 0.5000 & 0.5000 & 1.0000 & $0.02992(11)$ \\
N1 & $0.42504(18)$ & $0.4659(3)$ & $0.83182(15)$ & $0.0348(5)$ \\
N2 & $0.1196(2)$ & $0.5149(3)$ & $0.51529(17)$ & $0.0452(6)$ \\
01 & $0.69512(15)$ & $0.4641(3)$ & $1.01165(15)$ & $0.0491(5)$ \\
O2 & $0.70226(18)$ & $0.4950(3)$ & $0.85630(16)$ & $0.0602(7)$ \\
O3 & $1.11681(17)$ & $0.5634(3)$ & $0.85459(15)$ & $0.0595(6)$ \\
H3 & 1.1863 & 0.5732 & 0.8777 & $0.089^{*}$ \\
O4 & $0.49253(16)$ & $0.2294(2)$ & $1.02284(14)$ & $0.0468(5)$ \\
H4A & 0.4257 & 0.2014 & 1.0295 & $0.070^{*}$ \\
H4B & 0.5364 & 0.2033 & 1.0775 & $0.070^{*}$ \\
05 & $0.6671(3)$ & $0.3802(4)$ & $0.66682(19)$ & $0.0835(8)$ \\
H5A & 0.7024 & 0.4475 & 0.6386 & $0.125^{*}$ \\
H5B & 0.6757 & 0.4148 & 0.7252 & $0.125^{*}$ \\
\hline & $0.27584(16)$ & $0.4099(3)$ & $0.47317(13)$ & $0.0506(5)$ \\
\hline
\end{tabular}

half of a bpfp ligand [14-16], one half of a $\mathrm{L}^{2-}$ ligand, one coordinated water molecule and one solvent water molecule (see the figure). The $\mathrm{Cd}(\mathrm{II})$ centre is in a distorted octahedral geometry with the $\mathrm{CdO}_{4} \mathrm{~N}_{2}$ coordination. The $\mathrm{Cd}-\mathrm{O} / \mathrm{N}$ distances associated with central $\mathrm{Cd}(\mathrm{II})$ centres are in the range of 2.297(2)-2.335 (2) $\AA$ and the bond angles about the $\mathrm{Cd}(\mathrm{II})$ centers range from $78.02(7)$ to $180.0^{\circ}$. In the title compound, $\mathrm{L}^{2-}$ anions and bpfp ligands link $\mathrm{Cd}(\mathrm{II})$ centers to a two-dimensional structure.

Author contributions: All the authors have accepted responsibility for the entire content of this submitted manuscript and approved submission.
Research funding: This work was supported by the grants from the PhD Startup Fund of HAUST (13480061).

Conflict of interest statement: The authors declare no conflicts of interest regarding this article.

\section{References}

1. Agilent Technologies. CrysAlis ${ }^{P R O}$ Software System, version 1.171.38.41r; Agilent Technologies UK Ltd: Oxford, UK, 2015.

2. Sheldrick G. M. SHELXTL - integrated space-group and crystalstructure determination. Acta Crystallogr. 2015, A71, 3-8.

3. Sheldrick G. M. Crystal structure refinement with SHELXL. Acta Crystallogr. 2015, C71, 3-8.

4. Dolomanov O. V., Bourhis L. J., Gildea R. J., Howard J. A. K., Puschmann H. OLEX2: a complete structure solution, refinement and analysis program. J. Appl. Crystallogr. 2009, 42, 339-341.

5. Du X.-G., Mi G., Liu J.-C., Zhang J. A two-dimensional Zn(II) coordination polymer with a three-dimensional supramolecular architecture comprising 5-dimethylamino-isophthalic acid and 1,3-bis(4-pyridyl)propane. Mol. Cryst. Liq. Cryst. 2016, 624, 44-50.

6. Du X.-G., Mi G., Liu J.-C., Zhang J. A luminescent Zn(II) supramolecular coordination polymer constructed from 3,4-thiophenedicarboxylic acid and flexible benzimidazolebased connector. Chin. J. Struct. Chem. 2014, 33, 1597-1602.

7. Li Z.-H., Xue L.-P., Mu Y.-J., Zhao B.-T. Viologen-derived material showing photochromic, visually oxygen responsive, and photomodulated luminescence behaviors. CrystEngComm 2021, 23, 1019-1024.

8. Lin X.-M., Li T.-T., Chen L.-F., Zhang L., Su C.-Y. Two ligandfunctionalized $\mathrm{Pb}$ (II) metal-organic frameworks: structures and catalytic performances. Dalton Trans. 2012, 41, 10422-10429.

9. Jiang X., Li Z., Zhai Y., Yan G., Xia H., Li Z. Porous coordination polymers based on azamacrocyclic complex: syntheses, solventinduced reversible crystal-to-crystal transformation and gas sorption properties. CrystEngComm 2014, 16, 805-813.

10. Hao H.-J., Liu F.-J., Su H.-F., Wang Z.-H., Wang D.-F., Huang R.-B., Zheng L.-S. Syntheses, structures and fluorescence of two coordination complexes of $\mathrm{Zn}$ (II) and 1,3-bis(2-methylimidazolyl) propane: solvent effect. CrystEngComm 2012, 14, 6726-6731.

11. Li M., Ling Q., Yang Z., Li B.-L., Li H.-Y. Seven structural versatile coordination polymers based on a flexible bis(triazole) and polycarboxylate co-ligands: syntheses, structures and properties. CrystEngComm 2013, 15, 3630-3639.

12. Xue L. Crystal structure of catena-poly[diaqua- $\left(\mu_{2}-\right.$ 2,5-dihydroxyterephthalato- $\left.\kappa^{4} O, O^{\prime}: O^{\prime \prime}, O^{\prime \prime \prime}\right)$ cadmium(II)], $\mathrm{C}_{8} \mathrm{H}_{8} \mathrm{O}_{8}$ Cd. Z. Kristallogr. NCS 2018, 233, 271-272.

13. Wang Y. L., Jiang Y. L., Xiahou Z. J., Fu J. H., Liu Q. Y. Diversity of lanthanide (III)-2,5-dihydroxy-1,4-benzenedicarboxylate extended frameworks: syntheses, structures, and magnetic properties. Dalton Trans. 2012, 41, 11428-11437.

14. Wang C. Y., Wilseck Z. M., Supkowski R. M., LaDuca R. L. Metal and ligand binding mode dependent topologies in phthalate coordination polymers with bis(4-pyridylformyl)piperazine coligands. CrystEngComm 2011, 13, 1391-1399. 
15. Farnum G. A., Lucas J. S., Wang C. Y., LaDuca R. L. Luminescent cadmium and zinc diphenate coordination polymers containing pyridyl-piperazine type ligands: grids, diamondoid lattices, and a rare 4-connected net. Inorg. Chim. Acta. 2011, 368, 84-95.
16. Mizzi J. E., LaDuca R. L. Divalent metal pyromellitate coordination polymers containing bis(4-pyridylformyl)piperazine: selection between simple chain and rare fsc network topologies. Inorg. Chim. Acta. 2014, 421, 183-190. 